Table DR1. Foraminiferal oxygen and carbon isotope data for ODP Site 1050. Benthic foraminifera include species of Nuttalides, Oridorsalis, Gavelinella, Berthelina, and Gyroidina. All other species listed are planktic foraminifera. Stable isotope data expressed as per mil values relative to the Vienna Peedee belemnite standard.

\begin{tabular}{|c|c|c|c|c|c|}
\hline SAMPLE & MBSF & AGE & SPECIES & $\delta^{13} \mathrm{C}$ & $\delta^{18} \mathrm{O}$ \\
\hline 19R-1, 69-72 & 481.69 & 76.01 & Heterohelix globulosa & 2.471 & -0.526 \\
\hline 19R-1, 69-72 & 481.69 & 76.01 & Contusotruncana fornicata & 2.540 & -0.658 \\
\hline 19R-1, 69-72 & 481.69 & 76.01 & Nuttalides sp. & 1.762 & 0.101 \\
\hline 19R-1, 69-72 & 481.69 & 76.01 & Globotruncana elevata & 2.499 & -0.592 \\
\hline 19R-1, 69-72 & 481.69 & 76.01 & Globigerinelloides prairiehilliensis & 2.546 & -0.535 \\
\hline 19R-1, 69-72 & 481.69 & 76.01 & Pseudotextularia nuttalli & 2.881 & -0.520 \\
\hline 19R-2, 78-81 & 483.28 & 76.07 & Nuttalides sp. & 1.773 & -0.270 \\
\hline 19R-2, 78-81 & 483.28 & 76.07 & Contusotruncana fornicata & 2.513 & -0.580 \\
\hline 19R-2, 78-81 & 483.28 & 76.07 & Heterohelix globulosa & 2.401 & -0.653 \\
\hline 19R-2, 78-81 & 483.28 & 76.07 & Globigerinelloides prairiehilliensis & 2.473 & -0.522 \\
\hline 19R-2, 78-81 & 483.28 & 76.07 & Archaeoglobigerina blowi & 2.510 & -0.708 \\
\hline $19 R-3,15-18$ & 484.15 & 76.26 & Heterohelix globulosa & 2.522 & -0.613 \\
\hline 19R-3, 15-18 & 484.15 & 76.26 & Archaeoglobigerina blowi & 2.530 & -0.774 \\
\hline 19R-3, 15-18 & 484.15 & 76.26 & Contusotruncana fornicata & 2.444 & -0.799 \\
\hline $19 R-3,15-18$ & 484.15 & 76.26 & Nuttalides sp. & 1.787 & 0.154 \\
\hline $19 R-3,15-18$ & 484.15 & 76.26 & Pseudotextularia nuttalli & 2.841 & -0.363 \\
\hline 19R-3, 15-18 & 484.15 & 76.26 & Globigerinelloides prairiehilliensis & 2.581 & -0.720 \\
\hline $20 \mathrm{R}-1,100-101$ & 491.60 & 87.30 & Nuttalides sp. & 1.523 & -1.293 \\
\hline 20R-1, 100-101 & 491.60 & 87.30 & Marginotruncana pseudolinneiana & 2.452 & -1.241 \\
\hline 20R-1, 42-45 & 491.02 & 77.06 & Heterohelix globulosa & 2.648 & -0.574 \\
\hline 20R-1, 42-45 & 491.02 & 77.06 & Contusotruncana fornicata & 2.622 & -0.736 \\
\hline $20 \mathrm{R}-1,42-45$ & 491.02 & 77.06 & Nuttalides sp. & 1.794 & -0.113 \\
\hline 20R-2, 5-6 & 492.15 & 87.50 & Nuttalides sp. & 1.933 & -0.939 \\
\hline 20R-2, 5-6 & 492.15 & 87.50 & Praeglobotruncana gibba & 2.714 & -1.080 \\
\hline 20R-2, 5-6 & 492.15 & 87.50 & Marginotruncana pseudolinneiana & 2.630 & -1.490 \\
\hline 20R-2, 58-61 & 492.68 & 87.60 & Contusotruncana fornicata & 2.784 & -1.050 \\
\hline 20R-2, 58-61 & 492.68 & 87.60 & Heterohelix globulosa & 2.771 & -1.101 \\
\hline 20R-2, 58-61 & 492.68 & 87.60 & Marginotruncana pseudolinnelana & 2.652 & -1.035 \\
\hline $20 R-2,58-61$ & 492.68 & 87.60 & Nuttalides sp. & 1.763 & -0.897 \\
\hline 20R-3, 38-41 & 492.93 & 91.10 & Heterohelix globulosa & 2.965 & -1.628 \\
\hline 20R-3, 38-41 & 492.93 & 91.10 & Helvetoglobotruncana helvetica & 3.181 & -1.905 \\
\hline 20R-3, 38-41 & 492.93 & 91.10 & Helvetoglobotruncana helvetica & 2.712 & -1.755 \\
\hline 20R-3, 38-41 & 492.93 & 91.10 & Contusotruncana fornicata & 2.444 & -0.799 \\
\hline 20R-3, 38-41 & 492.93 & 91.10 & Praeglobotruncana gibba & 3.077 & -1.737 \\
\hline 20R-3, 38-41 & 492.93 & 91.10 & Oridorsalis sp. & 2.436 & -0.631 \\
\hline 20R-3, 76-77 & 494.31 & 91.24 & Nuttalides sp. & 2.135 & -0.757 \\
\hline 22R-1, 33-36 & 510.13 & 94.64 & Praeglobotruncana stephani & 1.989 & -2.389 \\
\hline 22R-1, 33-36 & 510.13 & 94.64 & Hedbergella simplex & 1.546 & -1.950 \\
\hline $22 \mathrm{R}-1,33-36$ & 510.13 & 94.64 & Globigerinelloides bentonensis & 1.833 & -2.370 \\
\hline $22 \mathrm{R}-1,33-36$ & 510.13 & 94.64 & Lenticulina sp. & 0.106 & -1.163 \\
\hline $22 \mathrm{R}-1,46-50$ & 510.26 & 94.65 & Berthelina sp. & 1.550 & -0.462 \\
\hline $22 \mathrm{R}-1,46-50$ & 510.26 & 94.65 & Whiteinella brittonensis & 2.163 & -2.626 \\
\hline $22 \mathrm{R}-1,46-50$ & 510.26 & 94.65 & Rotalipora greenhornensis & 2.162 & -1.610 \\
\hline $22 \mathrm{R}-1,46-50$ & 510.26 & 94.65 & Cibicides sp. & 1.303 & -0.738 \\
\hline
\end{tabular}




\begin{tabular}{|c|c|}
\hline $22 \mathrm{R}-1,46-50$ & 510.26 \\
\hline 23R-1, 4-5 & 519.44 \\
\hline 23R-1, 4-5 & 519.44 \\
\hline $23 R-1,4-5$ & 519.44 \\
\hline $23 R-1,4-5$ & 519.44 \\
\hline 23R-1, 4-5 & 519.44 \\
\hline 23R-2, 109-111 & 521.89 \\
\hline 23R-2, 109-111 & 521.89 \\
\hline 23R-2, 19-21 & 521.60 \\
\hline 23R-2, 19-21 & 521.60 \\
\hline 23R-2, 19-21 & 521.60 \\
\hline 23R-2, 48.5-49.5 & 521.39 \\
\hline 23R-2, 48.5-49.5 & 521.39 \\
\hline 23R-3, 127-130 & 523.67 \\
\hline 23R-3, 127-130 & 523.67 \\
\hline 23R-3, 127-130 & 523.67 \\
\hline 23R-3, 127-130 & 523.67 \\
\hline 23R-3, 131-134 & 523.71 \\
\hline 23R-3, 131-134 & 523.71 \\
\hline 23R-3, 131-134 & 523.67 \\
\hline $23 R-3,49.5-50.5$ & 522.90 \\
\hline 23R-3, 49.5-50.5 & 522.90 \\
\hline 23R-4, 60-64 & 524.50 \\
\hline 23R-4, 60-64 & 524.50 \\
\hline $23 R-4,60-64$ & 524.50 \\
\hline 23R-4, 60-64 & 524.50 \\
\hline 23R-5, 23-26 & 525.63 \\
\hline 23R-5, 23-26 & 525.63 \\
\hline 23R-5, 23-26 & 525.63 \\
\hline 23R-5, 23-26 & 525.63 \\
\hline 23R-6, 126-129 & 529.42 \\
\hline $23 R-6,126-129$ & 529.42 \\
\hline 24R-1, 32-35 & 531.91 \\
\hline 24R-1, 32-35 & 531.91 \\
\hline 24R-1, 32-35 & 531.91 \\
\hline $24 \mathrm{R}-1,32-35$ & 531.91 \\
\hline 24R-2, 69-72 & 531.29 \\
\hline 24R-2, 69-72 & 531.29 \\
\hline 24R-2, 69-72 & 531.29 \\
\hline 24R-2, 69-72 & 531.29 \\
\hline 24R-2, 69-72 & 531.29 \\
\hline 24R-3, 45-48 & 532.45 \\
\hline 24R-3, 45-48 & 532.45 \\
\hline 24R-3, 45-48 & 532.45 \\
\hline 25R-1, 82-85 & 539.52 \\
\hline 25R-1, 82-85 & 539.52 \\
\hline 25R-1, 82-85 & 539.52 \\
\hline 25R-2, 83-87 & 541.03 \\
\hline 25R-2, 83-87 & 541.03 \\
\hline $25 R-2,83-87$ & 541.03 \\
\hline
\end{tabular}

94.65 Whiteinella brittonensis

95.60 Rotalipora maslakovae

95.60 Hedbergella delrioensis

95.60 Rotalipora greenhornensis

95.60 Heterohelix moremani

95.60 mixed benthics

95.85 Berthelina sp.

95.85 Rotalipora globotruncanoides

95.82 Praeglobotruncana stephani

95.82 Gavelinella sp.

95.82 Hedbergella delrioensis

95.80 Rotalipora greenhornensis

95.80 Planulina sp.

96.04 Heterohelix moremani

96.04 Whiteinella brittonensis

96.04 Rotalipora greenhornensis

96.04 Berthelina sp.

96.04 Heterohelix moremani

96.04 Rotalipora greenhornensis

96.04 Berthelina sp.

95.96 Rotalipora greenhornensis

95.96 mixed benthics

96.12 Heterohelix moremani

96.12 Hedbergella delrioensis

96.12 Rotalipora greenhornensis

96.12 Planulina sp.

96.24 Rotalipora greenhornensis

96.24 Rotalipora greenhornensis

96.24 Heterohelix moremani

96.24 mixed benthics

96.63 Rotalipora greenhornensis

96.63 mixed benthics

96.89 Heterohelix moremani

96.89 Rotalipora greenhornensis

96.89 Hedbergella simplex

96.89 Berthelina sp.

96.82 Hedbergella simplex

96.82 Hedbergella delrioensis

96.82 Gavelinella sp.

96.82 Rotalipora appenninica

96.82 Praeglobotruncana stephani

96.94 Hedbergella delrioensis

96.94 Rotalipora greenhornensis

96.94 Berthelina sp.

97.67 Hedbergella delrioensis

97.67 Rotalipora gandolfii

97.67 Berthelina sp.

97.83 Gavelinella sp.

97.83

97.83
Rotalipora globotruncanoides

Praeglobotruncana stephani

$\begin{array}{ll}2.233 & -2.549 \\ 2.712 & -1.141 \\ 3.262 & -1.574 \\ 2.864 & -1.247 \\ 2.018 & -1.248 \\ 2.528 & 0.023 \\ 1.283 & -0.762 \\ 2.269 & -1.918 \\ 2.941 & -2.099 \\ 1.998 & 0.069 \\ 2.651 & -3.270 \\ 3.160 & -1.882 \\ 2.632 & -0.236 \\ 0.957 & -2.233 \\ 2.274 & -2.756 \\ 2.064 & -2.046 \\ 1.186 & -0.503 \\ 1.003 & -1.680 \\ 2.023 & -1.717 \\ 1.465 & -0.410 \\ 2.495 & -1.911 \\ 2.066 & -0.504 \\ 0.978 & -2.033 \\ 2.052 & -2.906 \\ 2.028 & -1.924 \\ 1.279 & -0.684 \\ 1.870 & -1.774 \\ 2.063 & -1.811 \\ 1.075 & -1.777 \\ 1.319 & -0.668 \\ 1.849 & -2.108 \\ 1.072 & -0.600 \\ 0.129 & -2.710 \\ 1.745 & -2.085 \\ 1.576 & -1.928 \\ 1.194 & -0.761 \\ 1.445 & -1.664 \\ 1.784 & -2.417 \\ 0.896 & -0.712 \\ 1.421 & -2.186 \\ 1.743 & -2.224 \\ 1.649 & -2.581 \\ 1.584 & -2.332 \\ 1.060 & -0.832 \\ 1.882 & -2.035 \\ 1.626 & -2.116 \\ 1.185 & -0.656 \\ 1.374 & -0.583 \\ 1.680 & -2.220 \\ 1.931 & -2.368\end{array}$


25R-2, 83-87

25R-3, 60-64

25R-3, 60-64

25R-3, 60-64

25R-4, 16-20

25R-4, 16-20

25R-4, 16-20

26R-1, 139-141

26R-1, 139-141

26R-1, 139-141

26R-1, 53-56

26R-1, 53-56

26R-1, 53-56

26R-1, 53-56

26R-1, 53-56

26R-1, 53-56

26R-1, 53-56

26R-2, 56-59

26R-2, 56-59

26R-2, 56-59

26R-2, 56-59

26R-3, 120-122

26R-3, 120-122

26R-3, 73-76

26R-3, 73-76

26R-3, 73-76

26R-4, 140-142

26R-4, 140-142

26R-4, 140-142

26R-4, 83-86

26R-4, 83-86

26R-4, 83-86

26R-4, 83-86

26R-4, 83-86

26R-4, 83-86

26R-5, 83-86

26R-5, 83-86

26R-5, 83-86

26R-CC, 9-10

26R-CC, 9-10

26R-CC, 9-10

27R-1, 73-76

27R-1, 73-76

27R-1, 73-76

27R-1, 73-76

27R-2, 85-88

27R-2, 85-88

27R-2, 85-88

27R-2, 85-88

27R-2, 85-88
541.03

542.30

542.30

542.30

543.36

543.36

543.36

549.69

549.69

549.69

548.93

548.93

548.93

548.93

548.93

548.93

548.93

550.46

550.46

550.46

550.46

552.60

552.60

552.13

552.13

552.13

554.30

554.30

554.30

553.73

553.73

553.73

553.73

553.73

553.73

555.23

555.23

555.23

555.57

555.57

555.57

558.73

558.73

558.73

558.73

560.35

560.35

560.35

560.35

560.35
97.83 Nuttalides sp.

97.96 Hedbergella delrioensis

97.96 Rotalipora gandolfii

97.96 Berthelina sp.

98.07 Rotalipora gandolfii

98.07 Hedbergella delrioensis

98.07 mixed benthics

98.73 Costellagerina libyca

98.73 Gavelinella sp.

98.73 Rotalipora gandolfii

98.65 Gavelinella sp.

98.65 Hedbergella simplex

98.65 Berthelina $s p$.

98.65 Praeglobotruncana stephani

98.65

98.65

98.65

98.81

98.81

98.81

98.81

99.03

99.03

98.98

98.98

98.98

99.20

99.20

99.20

99.14

99.14

99.14

99.14

99.14

99.14

99.30

99.30

99.30

99.33

99.33

99.33

99.66

99.66

99.66

99.66

99.83

99.83

99.83

99.83

99.83

\section{Costellagerina libyca}

Rotalipora gandolfii

Hedbergella delrioensis

Rotalipora gandolfii

Hedbergella delrioensis

Costellagerina libyca

Nuttalides sp.

Rotalipora spp.*

Berthelina spp. *

Rotalipora gandolfii

Costellagerina libyca

Berthelina sp.

Rotalipora spp. *

Costellagerina libyca*

Berthelina spp. *

Rotalipora spp.*

Costellagerina libyca*

Costellagerina libyca

Rotalipora appenninica

Berthelina spp. *

Gyroidina globosa

Rotalipora appenninica

Costellagerina libyca

Berthelina sp.

Gavelinella sp.

Costellagerina libyca

Rotalipora appenninica

Costellagerina libyca

Biticinella breggiensis

Gavelinella sp.

Rotalipora appenninica

Rotalipora spp. *

Rotalipora ticinensis

Biticinella breggiensis

Praeglobotruncana delrioensis

Berthelina sp.
$1.241 \quad 0.070$

$1.872-2.159$

$\begin{array}{ll}1.643 & -2.522\end{array}$

$0.861-0.705$

$1.532-2.221$

$1.932-2.148$

$1.501-0.749$

$2.120-2.593$

$1.450-0.722$

$1.681-2.340$

$0.675 \quad-0.646$

$1.626-1.911$

$0.194-0.717$

$1.789-2.581$

$1.924-3.230$

$1.639-2.511$

$2.074-2.606$

$1.674-2.112$

$2.041-2.354$

$2.734-3.088$

$1.035-0.549$

$1.499-2.258$

$0.077 \quad-0.507$

$1.720-1.784$

$2.005-2.385$

$0.889-0.586$

$2.180-2.441$

$2.498-2.896$

$-0.101 \quad-0.903$

$2.320-2.790$

$2.678-3.154$

$2.723 \quad-3.418$

$2.351-2.844$

$0.504-0.506$

$0.657-0.619$

$2.477 \quad-2.477$

$2.532-2.914$

$0.904-0.837$

$0.991-0.538$

$2.782-2.791$

$2.337-2.611$

$2.172-2.772$

$1.790-2.850$

$0.291-0.768$

$1.557-2.609$

$1.579-1.501$

$1.555-2.503$

$1.337-2.974$

$1.500-2.488$

$0.024-0.924$ 
27R-2, 85-88

27R-3, 79-82

27R-3, 79-82

27R-3, 79-82

27R-3, 79-82

27R-3, 79-82

27R-4, 134-136

27R-4, 134-136

27R-4, 134-136

27R-4, 34-36

27R-4, 34-36

27R-4, 34-36

27R-4, 80-83

27R-5, 123-125

27R-5, 32-34

27R-5, 32-34

27R-5, 32-34

27R-5, 32-34

27R-6, 74-78

27R-6, 74-78

27R-6, 74-78

27R-6, 74-78

27R-CC

27R-CC

27R-CC

28R-1, 70-73

28R-1, 70-73

28R-1, 70-73

28R-1, 70-73

28R-1, 20-21

28R-1, 20-21

28R-1, 20-21

28R-2, 61-64

28R-3, 60-63

28R-3, 60-63

28R-3, 60-63

28R-3, 60-63

28R-4, 146-1050

28R-4, 146-1050

28R-4, 146-1050

28R-4, 146-1050

28R-4, 66-69

28R-4, 66-69

28R-5, 50-53

28R-5, 50-53

28R-5, 50-53

28R-5, 50-53

28R-5, 50-53

29R-1, 57-60

29R-6, 21-24

\begin{tabular}{|c|c|c|}
\hline 560.35 & 99.83 & Berthelina spp. ${ }^{*}$ \\
\hline 561.79 & 99.98 & Rotalipora appenninica \\
\hline 561.79 & 99.98 & Costellagerina libyca \\
\hline 561.79 & 99.98 & Berthelina sp. \\
\hline 561.79 & 99.98 & Hedbergella simplex \\
\hline 561.79 & 99.98 & Berthelina sp. \\
\hline 563.84 & 99.96 & Hedbergella portsdownensis \\
\hline 563.84 & 99.96 & Rotalipora spp. * \\
\hline 563.84 & 99.96 & Berthelina spp. * \\
\hline 562.84 & 99.93 & Rotalipora spp. * \\
\hline 562.84 & 99.93 & Costellagerina libyca* \\
\hline 562.84 & 99.93 & Berthelina spp. * \\
\hline 563.30 & 99.94 & Planulina sp. \\
\hline 565.23 & 99.99 & Berthelina spp. * \\
\hline 564.32 & 99.97 & Biticinella breggiensis \\
\hline 564.32 & 99.97 & Rotalipora ticinensis \\
\hline 564.32 & 99.97 & Hedbergella simplex \\
\hline 564.32 & 99.97 & Berthelina sp. \\
\hline 566.24 & 100.02 & Hedbergella portsdownensis \\
\hline 566.24 & 100.02 & Rotalipora appenninica \\
\hline 566.24 & 100.02 & Berthelina sp. \\
\hline 566.24 & 100.02 & Berthelina sp. \\
\hline 567.56 & 100.06 & Rotalipora ticinensis \\
\hline 567.56 & 100.06 & Costellagerina libyca \\
\hline 567.56 & 100.06 & Biticinella breggiensis \\
\hline 568.30 & 100.08 & Berthelina sp. \\
\hline 568.30 & 100.08 & Biticinella breggiensis \\
\hline 568.30 & 100.08 & Rotalipora ticinensis \\
\hline 568.30 & 100.08 & Rotalipora ticinensis \\
\hline 567.80 & 100.07 & Rotalipora ticinensis \\
\hline 567.80 & 100.07 & Biticinella breggiensis \\
\hline 567.80 & 100.07 & Biticinella breggiensis \\
\hline 569.71 & 100.12 & Berthelina sp. \\
\hline 571.20 & 100.16 & Hedbergella delrioensis \\
\hline 571.20 & 100.16 & Berthelina sp. \\
\hline 571.20 & 100.16 & Biticinella breggiensis \\
\hline 571.20 & 100.16 & Biticinella breggiensis \\
\hline 573.56 & 100.23 & Hedbergella portsdownensis \\
\hline 573.56 & 100.23 & Berthelina sp. \\
\hline 573.56 & 100.23 & Rotalipora ticinensis \\
\hline 573.56 & 100.23 & Rotalipora ticinensis \\
\hline 572.76 & 100.20 & Rotalipora appenninica \\
\hline 572.76 & 100.20 & Planulina sp. \\
\hline 574.10 & 100.24 & Hedbergella simplex \\
\hline 574.10 & 100.24 & Berthelina sp. \\
\hline 574.10 & 100.24 & Hedbergella almadensis \\
\hline 574.10 & 100.24 & Biticinella breggiensis \\
\hline 574.10 & 100.24 & Berthelina flat \\
\hline 577.77 & 100.34 & Berthelina sp. \\
\hline 584.91 & 100.54 & Berthelina sp. \\
\hline
\end{tabular}

$\begin{array}{ll}0.688 & -0.697 \\ 1.807 & -2.067 \\ 2.127 & -2.475 \\ -0.012 & -0.852 \\ 1.839 & -2.435 \\ 0.291 & -0.297 \\ 1.860 & -1.129 \\ 1.731 & -1.663 \\ 0.420 & 0.150 \\ 1.692 & -2.942 \\ 1.126 & -2.465 \\ -0.196 & -0.810 \\ 0.468 & -0.727 \\ 0.728 & -0.214 \\ 1.827 & -2.817 \\ 1.672 & -2.315 \\ 1.544 & -2.179 \\ 0.111 & -0.528 \\ 1.789 & -2.454 \\ 1.753 & -2.256 \\ 0.061 & -0.597 \\ 0.137 & -0.621 \\ 1.513 & -2.267 \\ 2.560 & -3.400 \\ 1.409 & -2.866 \\ 0.260 & 0.142 \\ 1.600 & -2.526 \\ 1.357 & -1.430 \\ 1.733 & -2.340 \\ 1.332 & -1.353 \\ 1.508 & -2.211 \\ 1.491 & -3.219 \\ 0.420 & -0.626 \\ 1.952 & -2.636 \\ -0.320 & -0.946 \\ 1.599 & -3.019 \\ 1.589 & -2.936 \\ 1.770 & -2.706 \\ -0.279 & -0.813 \\ 1.634 & -2.579 \\ 1.463 & -2.222 \\ 1.452 & -1.206 \\ 0.422 & -0.717 \\ 1.640 & -2.145 \\ 0.018 & -0.707 \\ 1.799 & -2.934 \\ 1.568 & -3.136 \\ 0.472 & -0.639 \\ 0.017 & -0.490 \\ 0.460 & -0.640\end{array}$




29R-6, 21-24
29R-6, 21-24
29R-6, 21-24
29R-5, 72-75
29R-5, 72-75
29R-5, 72-75
29R-5, 72-75
30R-2, 72-75
30R-2, 72-75
30R-2, 72-75
30R-3, 72-75
30R-3, 72-75
30R-4, 78-81
30R-4, 78-81
30R-4, 78-81
31R-1, 81-85
31R-1, 81-85
31R-1, 81-85
31R-2, 80-84
31R-2, 80-84
31R-3, 50-53
31R-3, 50-53
31R-3, 50-53
31R-4, 87-90
31R-4, 87-90
31R-4, 87-90
31R-5, 81-84
31R-5, 81-84
31R-5, 81-84
31R-6, 79-83
31R-6, 79-83
31R-6, 79-83
31R-CC
31R-CC
31R-CC

584.91

584.91

584.91

583.92

583.92

583.92

583.92

589.02

589.02

589.02

590.52

590.52

592.08

592.08

592.08

597.21

597.21

597.21

598.70

598.70

600.20

600.20

600.20

601.77

601.77

601.77

603.21

603.21

603.21

604.69

604.69

604.69

606.00

606.00

606.00
100.54 Rotalipora ticinensis

100.54 Biticinella breggiensis

100.54 Berthelina sp.

100.51 Epistomina sp.

100.51 Rotalipora ticinensis

100.51 Biticinella breggiensis

100.51 Berthelina sp.

100.65 Rotalipora ticinensis

100.65 Biticinella breggiensis

100.65 Epistomina sp.

100.70 Rotalipora ticinensis

100.70 Epistomina sp.

100.74 Rotalipora ticinensis

100.74 Biticinella breggiensis

100.74 Berthelina intermedia

100.88 Rotalipora ticinensis

100.88 Biticinella breggiensis

100.88 Berthelina sp.

100.92 Biticinella breggiensis

100.92 Hedbergella simplex

100.96 Ticinella primula

100.96 Globigerinelloides bentonensis

100.96 Bethelina sp.

101.01 Rotalipora ticinensis

101.01 Biticinella breggiensis

101.01 Berthelina sp.

101.05 Rotalipora ticinensis

101.05 Biticinella breggiensis

101.05 Ticinella primula

101.09 Rotalipora ticinensis

101.09 Hedbergella planispira

101.09 Berthelina sp.

101.12 Biticinella breggiensis

101.12 Rotalipora ticinensis

101.12 Berthelina sp.

$\begin{array}{ll}1.559 & -2.150 \\ 1.718 & -2.891 \\ 0.257 & -0.464 \\ 1.701 & -0.229 \\ 1.912 & -2.277 \\ 1.777 & -2.861 \\ -0.199 & -0.380 \\ 1.842 & -2.259 \\ 2.106 & -2.986 \\ 1.786 & -0.380 \\ 1.918 & -2.185 \\ 1.777 & -0.299 \\ 1.949 & -2.480 \\ 1.592 & -3.714 \\ 0.149 & -0.509 \\ 1.773 & -1.897 \\ 1.946 & -2.633 \\ -0.009 & -0.328 \\ 1.811 & -2.655 \\ 1.167 & -1.883 \\ 1.397 & -3.692 \\ 1.633 & -2.655 \\ -0.065 & -0.520 \\ 1.466 & -2.280 \\ 1.983 & -2.570 \\ -0.948 & -0.727 \\ 1.296 & -1.893 \\ 1.234 & -2.990 \\ 1.317 & -2.495 \\ 1.890 & -2.104 \\ 1.752 & -2.170 \\ 0.256 & -0.660 \\ 1.943 & -2.628 \\ 0.907 & -1.971 \\ 1.594 & -1.540\end{array}$


Table DR2. Foraminiferal oxygen and carbon isotope data for ODP Site 1049. Benthic foraminifera include species of Nuttalides and Gavelinella. All other species listed are planktic foraminifera. Stable isotope data expressed as per mil values relative to the Vienna Peedee belemnite standard.

\begin{tabular}{llcc} 
SAMPLE & SPECIES & $\delta^{13} \mathbf{C}$ & $\delta^{18} \mathbf{O}$ \\
\hline $11 X-01,100-102$ & Globotruncana linneiana & 2.127 & -0.381 \\
$11 X-01100-102$ & Rugoglobigerina rugosa & 2.237 & -0.323 \\
$11 X-01,100-102$ & Nuttalides sp. & 1.491 & 0.461 \\
$11 X-01,100-102$ & Heterohelix globulosa & 2.029 & -0.520 \\
$11 X-01,100-102$ & Radotruncana calcarata & 2.393 & -0.863 \\
$11 X-01,100-102$ & Contusotruncana fornicata & 2.583 & -0.490 \\
$11 X-01,100-102$ & Pseudotextuaria nuttalli & 2.919 & -0.527 \\
$11 X-01,50-52$ & Nuttalides sp. & 1.481 & 0.590 \\
$11 X-01,50-52$ & Heterohelix globulosa & 2.129 & -0.477 \\
$11 X-01,50-52$ & Contusotruncana fornicata & 2.803 & -0.639 \\
$11 X-01,50-52$ & Rugoglobigerina rugosa & 2.316 & -1.331 \\
$11 X-02,25-27$ & Nuttalides sp. & 1.586 & 0.253 \\
$11 X-02,25-27$ & Heterohelix globulosa & 1.961 & -0.275 \\
$11 X-02,25-27$ & Contusotruncana fornicata & 2.665 & -0.383 \\
$11 X-02,105-107$ & Nuttalides sp. & 1.587 & -0.299 \\
$11 X-02,105-107$ & Rugoglobigerina rugosa & 2.761 & -0.885 \\
$11 X-02,105-107$ & Pseudotextularia sp. & 2.617 & -0.425
\end{tabular}


Table DR3. Foraminiferal oxygen and carbon isotope data for DSDP Site 463. Benthic foraminifera include species of Nuttalides and Gavelinella. All other species listed are planktic foraminifera. All other species listed are planktic foraminifera. Stable isotope data expressed as per mil values relative to the Vienna Peedee belemnite standard.

\begin{tabular}{llcc} 
SAMPLE & SPECIES & $\mathbf{S}^{13} \mathbf{C}$ & $\boldsymbol{\delta}^{\mathbf{1 8}} \mathbf{O}$ \\
\hline 22R-03, 74-76 & Nuttalides sp. & 0.768 & -0.079 \\
22R-03, 74-76 & Radotruncana subspinosa & 2.136 & -1.279 \\
22R-03, 74-76 & Rugoblobotruncana subcircumnodifer & 2.721 & -1.110 \\
22R-03, 74-76 & Radotruncana calcarata & 2.197 & -1.396 \\
22R-03, 74-76 & Globigerinelloides prairiehillensis & 2.641 & -1.484 \\
22R-03, 74-76 & Heterohelix globulosa & 3.078 & -1.371 \\
22R-04, 74-76 & Heterohelix globulosa & 2.438 & -1.476 \\
22R-04, 74-76 & Radotruncana subspinosa & 1.973 & -1.903 \\
22R-04, 74-76 & Contusotruncana fornicata & 2.451 & -1.040 \\
22R-04, 74-76 & Radotruncana calcarata & 2.374 & -1.203 \\
22R-04, 74-76 & Globigerinelloides prairiehillensis & 2.469 & -1.351 \\
22R-04, 74-76 & Ruglobigerina rugosa & 2.227 & -1.701 \\
22R-04, 74-76 & Nuttalides sp. & 0.654 & 0.243 \\
26R-01, 74-76 & Nuttalides sp. & 1.003 & -0.106 \\
26R-01, 74-76 & Contusotruncana fornicata & 2.218 & -0.864 \\
26R-01, 74-76 & Heterohelix globulosa & 2.419 & -1.668 \\
26R-01, 74-76 & Rugoglobigerina rugosa & 2.195 & -1.004 \\
26R-05, 74-76 & Gavelinella sp. & 1.502 & -0.479 \\
26R-05, 74076 & Heterohelix globulosa & 2.458 & -1.396 \\
26R-05, 74-76 & Contusotruncana fornicata & 2.349 & -1.374 \\
34R-01, 79-81 & Gavelinella sp. & 2.298 & -1.390 \\
34R-01, 79-81 & Whiteninalla brittonensi & 2.867 & -2.251 \\
34R-02, 72-75 & Whiteinella brittonensis & 2.984 & -2.228 \\
35R-01, 17-19 & Gavelinella sp. & 2.092 & -1.614
\end{tabular}


Table DR4. Foraminiferal oxygen and carbon isotope data for DSDP Site 305. Benthic foraminifera include species of Nuttalides and Oridorsalis. All other species listed are planktic foraminifera. All other species listed are planktic foraminifera. Stable isotope data expressed as per mil values relative to the Vienna Peedee belemnite standard.

\begin{tabular}{llcc} 
SAMPLE & SPECIES & $\boldsymbol{\delta}^{13} \mathbf{C}$ & $\boldsymbol{\delta}^{18} \mathbf{O}$ \\
\hline 23R-02, 82-84 & Oridorsalis sp. & 0.995 & 0.685 \\
23R-02, 82-84 & Heterohelix globulosa & 3.086 & -0.841 \\
23R-02, 82-84 & Radotruncana subspinosa & 2.379 & -1.145 \\
23R-02, 82-84 & Radotrucana calcarata & 2.445 & -1.034 \\
23R-02, 82-84 & Globotruncana linneiana & 2.837 & -0.747 \\
23R-02, 82-84 & Rugoglobigerina rugosa & 2.863 & -0.750 \\
23R-02, 82-84 & Globgerinelloides prairiehillensis & 3.112 & -0.794 \\
23R-02, 82-84 & Globotruncan elevata & 2.731 & -0.765 \\
42R-01, 100-102 & Nuttalides sp. & 0.683 & -0.709 \\
42R-01, 100-102 & Hedbergella delrioensis & 1.699 & -3.076 \\
42R-1, 100-102 & Nuttalides sp. & 1.474 & -2.435
\end{tabular}




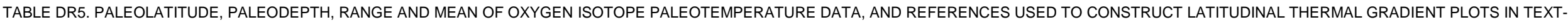

\begin{tabular}{|c|c|c|c|c|c|c|c|c|c|c|c|c|c|}
\hline $\begin{array}{l}\text { DSDP/ODP } \\
\text { Site } \\
\end{array}$ & Location & Paleolat. & Paleodepth & Foraminifer & a $104-102 \mathrm{Ma} \delta^{18} \mathrm{C}$ & $\begin{array}{c}\text { 104-102 Ma } \\
\text { Avg. Adj. Temp. }{ }^{*}\end{array}$ & $94-92 \mathrm{Ma} \delta^{18} \mathrm{O}$ & $\begin{array}{l}\text { 94-92 Ma Avg. } \\
\text { Adj. Temp.* }\end{array}$ & $75.5-74.5 \delta^{18} \mathrm{O}$ & $\begin{array}{r}\text { 75.5-74.5 Ma } \\
\text { Avg. Adj. Temp. }\end{array}$ & ${ }^{*} 68.5-66.5 \delta^{18} \mathrm{O}$ & $\begin{array}{l}\text { 68.5-66.5 Ma Avg } \\
\text { Adj. Temp.* }\end{array}$ & REFERENCE \\
\hline 144 & North Atlantic & $5^{\circ} \mathrm{N}$ & --- & planktic & -3.24 & & -3.55 to -4.09 & $33.4(n=19)$ & -3.93135 & 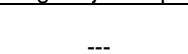 & 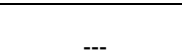 & (2) & Sellwood et al., 1994; \\
\hline 152 & & $9^{\circ} \mathrm{N}$ & --- & planktic & --- & (-) & - & $0.4(10-10)$ & -4.094 & --. & 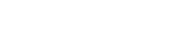 & 然 & Boersma, 1984 \\
\hline 152 & & $9^{\circ} \mathrm{N}$ & $\sim 1000 \mathrm{~m}$ (this study) & benthics & --- & --- & --- & --- & -3.553 & --- & & & Boersma, 1984 \\
\hline 551 & North Atlantic & $43^{\circ} \mathrm{N}$ & $\sim 2000$ to $3000 \mathrm{~m}$ (de & planktic & --- & --- & -2.24 to -2.44 & $25.0(n=4)$ & --- & --- & --- & --- & MacLeod et al., 2000 \\
\hline 551 & North Atlantic & $43^{\circ} \mathrm{N}$ & Graciansky, et al., 1985) & benthics & --- & --- & -1.21 to -1.74 & $19.3(n=3)$ & --- & --- & --- & --- & $\begin{array}{l}\text { MacLeod et al., } 2000 \\
\text { Boersma, 1984; Barrera \& }\end{array}$ \\
\hline 384 & North Atlantic & $33^{\circ} \mathrm{N}$ & $\sim 3000$ & planktic & --- & --- & --- & --- & --- & --- & -0.92 to -0.93 & $17.8(\mathrm{n}=2)$ & $\begin{array}{l}\text { Savin, } 1999 \\
\text { Boersma, 1984; Barrera \& }\end{array}$ \\
\hline 384 & North Atlantic & $33^{\circ} \mathrm{N}$ & (Tuchulke \& Vogt, 1979) & benthics & --- & --- & --- & --- & --- & --- & 0.18 to 0.71 & $10.3(n=10)$ & $\begin{array}{l}\text { Savin, } 1999 \\
\text { MacLeod et al., 2000; This }\end{array}$ \\
\hline $390 / 1049$ & North Atlantic & $30^{\circ} \mathrm{N}$ & $\sim 1400$ to $2000 \mathrm{~m}$ & planktic & --- & --- & --- & --- & -0.863 & $19.2(n=1)$ & -1.10 to -1.44 & $19.7(n=6)$ & $\begin{array}{l}\text { study } \\
\text { MacLeod et al., 2000; This }\end{array}$ \\
\hline $390 / 1049$ & North Atlantic & $30^{\circ} \mathrm{N}$ & (Norris et al., 1998) & benthics & --- & --- & --- & --- & 0.46 & $10.5(n=1)$ & 0.29 to 0.71 & 10.10 & $\begin{array}{l}\text { study } \\
\text { Huber et al., 1999, } \\
\text { MacLeod et al., 2000; This }\end{array}$ \\
\hline 1050 & North Atlantic & $30^{\circ} \mathrm{N}$ & --- & planktic & -2.87 to -3.34 & $28.9(n=6)$ & -1.62 to -2.62 & $24.6(n=18)$ & --- & --- & -1.39 to -1.94 & $20.5(n=5)$ & $\begin{array}{l}\text { study } \\
\text { Huber et al., 1999; }\end{array}$ \\
\hline 1050 & North Atlantic & $30^{\circ} \mathrm{N}$ & $\begin{array}{l}\sim 1100 \text { to } 2000 \mathrm{~m} \\
\text { (Norris et al., 1998) }\end{array}$ & benthics & 0.15 to -0.92 & $15.3(n=12)$ & -0.82 to -1.24 & $18.1(n=17)$ & --- & --- & 0.78 to 0.19 & $10.7(n=4)$ & $\begin{array}{l}\text { MacLeod et al., 2000; This } \\
\text { study } \\
\text { Wilson and Norris, 1998, }\end{array}$ \\
\hline 1052 & North Atlantic & $30^{\circ} \mathrm{N}$ & $\sim 200$ to 1000 & planktic & -2.67 to -3.73 & $-3.07(n=22)$ & --- & --- & -- & --- & --- & --- & $\begin{array}{l}\text { unpublished } \\
\text { Wilson and Norris, 1998, }\end{array}$ \\
\hline 1052 & North Atlantic & $30^{\circ} \mathrm{N}$ & (Norris et al., 1998) & benthics & -0.85 to -1.98 & $-1.45(n=18)$ & --- & --- & --- & --- & --- & --- & unpublished \\
\hline 356 & South Atlantic & $33^{\circ} \mathrm{S}$ & --- & planktic & --- & --- & --- & --- & --- & --- & -1.89 to -2.21 & $22.6(n=3)$ & Barrera and Savin, 1999 \\
\hline 356 & South Atlantic & $33^{\circ} \mathrm{S}$ & 2000-2500 (Sliter, 1977) & benthics & --- & --- & --- & --- & --- & --- & 0.14 to -1.14 & $14.2(n=8)$ & Barrera and Savin, 1999 \\
\hline 528 & South Atlantic & $38^{\circ} \mathrm{S}$ & 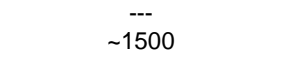 & planktic & --- & --- & --- & --- & --- & --- & -1.01 to -2.01 & $18.4(n=10)$ & D'Hondt \& Lindinger, 1994 \\
\hline 528 & South Atlantic & $38^{\circ} \mathrm{S}$ & (Moore et al., 1984) & benthics & --- & --- & --- & --- & --- & --- & 0.04 to -0.52 & $13.0(n=6)$ & D'Hondt \& Lindinger, 1994 \\
\hline 327 & South Atlantic & $58^{\circ} \mathrm{S}$ & $\sim 250$ to 1000 & planktic & --- & --- & --- & --- & $\begin{array}{c}-0.71 \text { to }-1.25 \\
-0.14\end{array}$ & $16.3(n=2)$ & --- & --- & Huber et al., 1995 \\
\hline 327 & South Atlantic & $58^{\circ} \mathrm{S}$ & (Sliter, 1977) & benthics & --- & --- & --- & --- & & $13.1(n=1)$ & --- & --- & Huber et al., 1995 \\
\hline 511 & South Atlantic & $58-60^{\circ} \mathrm{S}$ & $\sim 250$ to 1000 & planktic & -0.71 to -1.30 & $16.2(n=5)$ & -0.97 to -3.95 & $28.2(n=8)$ & 0.06 to -0.17 & $12.8(n=5)$ & --- & --- & Huber et al., 1995 \\
\hline 511 & South Atlantic & $58-60^{\circ} \mathrm{S}$ & (Sliter, 1977) & benthics & 0.30 to -0.42 & $12.5(n=9)$ & -0.30 to -1.26 & $19.2(n=2)$ & -1.40 to -1.83 & $19.0(n=9)$ & --- & --- & $\begin{array}{l}\text { Huber et al., } 1995 \\
\text { Barrera and Huber, 1990; }\end{array}$ \\
\hline 689 & South Atlantic & $67^{\circ} \mathrm{S}$ & $\sim 1000$ & planktic & --- & --- & --- & --- & -0.07 to -0.20 & $13.1(n=)$ & 0.55 to 0.46 & $9.1(n=3)$ & $\begin{array}{l}\text { Barrera and Savin, } 1999 \\
\text { Barrera and Huber, 1990; }\end{array}$ \\
\hline 689 & South Atlantic & $67^{\circ} \mathrm{S}$ & (Thomas, 1990) & benthics & --- & --- & --- & --- & 0.21 to 0.12 & $11.8(n=2)$ & 0.74 to 0.51 & $9.5(n=4)$ & $\begin{array}{l}\text { Barrera and Savin, } 1999 \\
\text { Barrera and Huber, 1990; }\end{array}$ \\
\hline 690 & South Atlantic & $68^{\circ} \mathrm{S}$ & $\sim 1800$ & planktic & --- & --- & --- & --- & -0.62 & $14.2(n=1)$ & 0.49 to -0.10 & $9.35(n=15)$ & $\begin{array}{l}\text { Barrera and Savin, } 1999 \\
\text { Barrera and Huber, 1990; }\end{array}$ \\
\hline 690 & South Atlantic & $68^{\circ} \mathrm{S}$ & (Thomas, 1990) & benthics & --- & --- & --- & --- & 0.03 to -0.06 & $12.6(n=2)$ & 0.72 to 0.47 & $9.68(n=5)$ & $\begin{array}{l}\text { Barrera and Savin, } 1999 \\
\text { Barrera and Savin, 1999; }\end{array}$ \\
\hline 305 & eq. Pacific & $2-7 \mathrm{~N}$ & $\sim 1000$ to 1500 & planktic & -3.08 & $30.1(n=1)$ & --- & --- & -1.03 to -1.15 & $20.6(n=3)$ & -1.10 to -1.14 & $18.2(n=3)$ & $\begin{array}{l}\text { This study } \\
\text { Barrera and Savin, 1999; }\end{array}$ \\
\hline 305 & eq. Pacific & $2-7^{\circ} \mathrm{N}$ & (this study) & benthics & -0.71 & $15.7(n=2)$ & --- & --- & 0.69 & $9.5(n=1)$ & 0.40 to 0.56 & 10.2 & $\begin{array}{l}\text { This study } \\
\text { Barrera and Savin, 1999; }\end{array}$ \\
\hline 463 & eq. Pacific & $12-8^{\circ} \mathrm{S}$ & $\sim 1500$ & planktic & --- & --- & -2.23 to -2.25 & $26.21(n=2)$ & -1.40 to -1.90 & $23.3(n=4)$ & -1.45 to -1.73 & $21.3(n=2)$ & $\begin{array}{l}\text { This study } \\
\text { Barrera and Savin, 1999; }\end{array}$ \\
\hline 463 & eq. Pacific & $12-8^{\circ} \mathrm{S}$ & (Boersma, 1981) & benthics & --- & --- & -1.39 to -1.64 & $19.3(n=2)$ & 0.24 to -0.08 & $12.1(\mathrm{n}=2)$ & 0.24 to 0.85 & $11.1(n=8)$ & This study \\
\hline 258 & so. Indian & $58^{\circ} \mathrm{S}$ & $\sim 1000$ to 1500 & planktic & -1.85 & $20.3(n=1)$ & --- & --- & --- & --- & --- & --- & Huber et al., 1995 \\
\hline 258 & so. Indian & $58^{\circ} \mathrm{S}$ & (this study) & benthics & -0.8 & $16.1(n=1)$ & --- & --- & --- & --- & --- & --- & Huber et al., 1995 \\
\hline
\end{tabular}

${ }^{*}$ Planktic model temperatures calculated using the palo

species and are adjusted for latitudinal salinity differences using the polynomial expression published by Zachos et al. (1994) 
Table DR6. Age model used for oxygen isotope data from ODP Site 1050.

\begin{tabular}{|c|c|c|c|c|}
\hline DATUM & $\begin{array}{c}1050 C \\
\text { Depth }^{1-5}\end{array}$ & $\begin{array}{c}\text { Planktic } \\
\text { Foraminifer Ages } \\
(\mathrm{Ma})^{6-8}\end{array}$ & $\begin{array}{c}\text { Calcareous } \\
\text { Nannofossil Ages } \\
(\mathrm{Ma})^{1,4}\end{array}$ & $\begin{array}{c}\text { Magnetic } \\
\text { Polarity Ages } \\
(\mathrm{Ma})^{9} \\
\end{array}$ \\
\hline $\mathrm{K} / \mathrm{T}$ boundary & 405.93 & & & \\
\hline base $P$. hariaensis & 412.96 & 66.0 & & \\
\hline base C30R & 426.86 & & & 67.8 \\
\hline base $M$. prinsii & 427.00 & & 66.0 & \\
\hline base $M$. murus & 444.70 & & 68.5 & \\
\hline base $A$. mayaroensis & 450.45 & 68.6 & & \\
\hline top C31R & 453.00 & & & 68.8 \\
\hline top C32n.1n & 455.30 & & & 71.0 \\
\hline base $R$. fructicosa & 456.29 & 69.5 & & \\
\hline top C32n.1r & 471.74 & & & 71.3 \\
\hline top C32n.2n & 477.56 & & & 71.7 \\
\hline top T. phacelosus & 478.00 & & 71.6 & \\
\hline base $P$. acervulinoides & 478.86 & 72.7 & & \\
\hline top $A$. parcus & 478.90 & & 74.6 & \\
\hline Top G. ventricosa Zone & 481.69 & 76.0 & & \\
\hline top E. eximius & 482.30 & & 75.0 & \\
\hline base $Q$. sissinghii & 491.40 & & 77.1 & \\
\hline base $C$. aculeus & 491.40 & & 78.5 & \\
\hline top L. septinarius & 491.40 & & 84.9 & \\
\hline base $G$. ventricosa & 491.68 & 79.8 & & \\
\hline base G. elevata & 491.68 & 83.2 & & \\
\hline top $D$. concavata & 492.15 & 85.3 & & \\
\hline base $D$. concavata & 492.68 & 90.3 & & \\
\hline top C34N & 492.77 & & & 83.5 \\
\hline top $H$. helvetica & 492.93 & 91.1 & & \\
\hline base A. parcus parcus & 493.10 & & 82.5 & \\
\hline base $E$. eximius & 493.70 & & 91.0 & \\
\hline base $M$. decussata & 495.40 & & 87.2 & \\
\hline base $H$. helvetica & 500.76 & 93.1 & & \\
\hline base $Q$. gartneri & 500.90 & & 93.5 & \\
\hline top C. kennedyi & 500.90 & 93.9 & & \\
\hline base $R$. riecheli & 523.65 & 95.9 & & \\
\hline base $C$. kennedyi & 529.30 & & 97.4 & \\
\hline base $C$. kennedyi & 544.10 & & 97.4 & \\
\hline base R. globotruncanoides & 551.03 & 98.9 & & \\
\hline top P. buxtorfi & 555.57 & 99.0 & & \\
\hline top B. breggiensis & 558.69 & 100.0 & & \\
\hline base $P$. buxtorfi & 562.84 & 100.0 & & \\
\hline base $R$. appenninica & 567.65 & 100.4 & & \\
\hline top T. praeticinensis & 574.10 & 100.3 & & \\
\hline top $R$. irregularis & 577.40 & & 99.0 & \\
\hline top $R$. irregularis & 586.70 & & 99.0 & \\
\hline
\end{tabular}

${ }^{1}$ Norris et al., 1998

${ }^{2}$ This study

${ }^{3}$ Ogg et al., 2000

${ }^{4}$ Self-Trail, 2001

${ }^{5}$ Huber et al., 1999

${ }^{6}$ Robaszynski \& Caron, 1995

${ }^{7}$ Bralower et al., 1998

${ }^{8}$ Premoli Silva and Sliter, 1995

${ }^{9}$ Kent and Gradstein, 1995 


\section{TABLE DR7. AGE MODEL USED FOR OXYGEN ISOTOPE DATA FROM DSDP SITE 511}

\begin{tabular}{|c|c|c|c|c|c|c|}
\hline Datum & Sample & Depth & Sr Isotope $e^{1,2}$ & $\begin{array}{c}\text { Planktic } \\
\text { Foraminifera }^{2} \\
\end{array}$ & $\begin{array}{c}\text { Calcareous } \\
\text { Nannofossils }\end{array}$ & Paleomagnetics $^{4}$ \\
\hline$\overline{\mathrm{Sr} \text { isotopes }}$ & $23-1$ & $\overline{195.38}$ & 71.8 & & & \\
\hline LAD G. impensus & $24-1,20-23$ & 204.70 & & 74.8 & & \\
\hline top C34N & & 225.00 & & & & 83.5 \\
\hline b A. parcus parcus & $30-2,70$ & 245.00 & & & 82.5 & \\
\hline t L. septinarius & $43-1,35$ & 366.00 & & & 84.9 & \\
\hline b M. decussata & $46-4,40$ & 399.00 & & & 87.2 & \\
\hline b M. furcatus & $46-4,40$ & 399.00 & & & 89.3 & \\
\hline b E. eximius & $48-1,40$ & 414.00 & & & 91.0 & \\
\hline b. E. turriseiffeli & $50-1,100$ & 434.00 & & & 101.7 & \\
\hline b A. albianus & $51-4,58$ & 447.00 & & & 106.5 & \\
\hline t $T$. bejaouaensis & $56-2,23-25$ & 491.23 & 112.5 & & & \\
\hline b T. bejaouaensis & $56-2,23-25$ & 491.23 & 114.0 & & & \\
\hline b. H. albiensis & $51, \mathrm{cc}$ & 451.00 & & & 114.0 & \\
\hline b Eiffellithus spp. & $55-5,32$ & 486.00 & & & 103.8 & \\
\hline b. S. achylosum & $57-4,43$ & 504.00 & & & 118.2 & \\
\hline
\end{tabular}

Bralower et al.. 1997; ${ }^{2}$ Huber et al., 1995; ${ }^{3}$ Wise, 1981; ${ }^{4}$ Salloway, 1981 
TABLE DR8. SURFACE- AND DEEP-WELLING PLANKTONIC FORAMINIFERA INCLUDED IN TEXT-FIGURE 2. DEPTH HABITATS INFERRED FROM STABLE ISOTOPIC DATA IN TABLES DR1-DR4

Surface dwelling taxa

Archaeoglobigerina blowi

Biticinella breggiensis

Costellagerina libyca

Helvetoglobotruncana helvetica

Radotruncana calcarata

Rugoglobigerina rugosa

Ticinella primula

Whiteinella brittonensis

Hedbergella delrioensis
Deep dwelling taxa

Contusotruncana fornicata

Globigerinelloides subcarinatus

Globotruncana linneiana

Hedbergella portsdownensis

Hedbergella simplex

Rotalipora appenninica

Rotalipora gandolfii

Rotalipora globotruncanoides

Rotalipora greenhornensis

Rotalipora ticinensis 


\section{TABLE DR9. REFERENCES CITED IN TABLE DR5}

Barrera, E., and Savin, S.M., 1999, Evolution of late Campanian-Mastrichtian marine climates and oceans, in Barrera, E. and Johnson, C., eds., Evolution of the Cretaceous Ocean-Climate System: Boulder, CO, Geological Society of America Special Paper 332, p. 245-282.

Boersma, A., 1981, Cretaceous-Tertiary foraminifers from Deep Sea Drilling Project Leg 62 sites in the Central Pacific, in Theide, J., Vallier, T. L., et al., Initial Reports of the Deep Sea Drilling Project, Volume 62: Washington, D.C., U.S. Govt. Printing Office, p. 377-396.

Boersma, A., 1984, Campanian through Paleocene paleotemperature and carbon isotope sequence and the Cretaceous-Tertiary boundary, Berggren, W. A. and Van Couvering, J. A., Catastrophes and Earth History Princeton, Princeton University Press, p. 247-277.

Boersma, A., 1984, Cretaceous-Tertiary planktonic foraminifers from the southeastern Atlantic, Walvis Ridge area, Deep Sea Drilling Project Leg 74, Moore Jr., T. C., Rabinowitz, P. D., et al., Initial Reports of the Deep Sea Drilling Project, Volume 74: Washington, D.C., U. S. Government Printing Office, p. 501-523.

D'Hondt, S., and Lindinger, M., 1994, A Maestrichtian stable isotopic record and its paleoceanographic implications: South Atlantic DSDP Site 528: Palaeogeography, Palaoeclimatology, Palaeoecology, v. 112, p. 363-378.

Graciansky, P.C. de, Poag, C.W., et al., 1985, Initial Reports of the Deep Sea Drilling Project, Volume 80: Washington, D.C., U.S. Government Printing Office, p. 1-679.

MacLeod, K.G., Huber, B.T., and Ducharme, M.L., 2000, Paleontological and geochemical constraints on changes in the deep ocean during the Cretaceous greenhouse interval, in Huber, B. T., MacLeod, K. G., and Wing, S. L., eds., Warm Climates in Earth History: Cambridge, Cambridge University Press, p. 241-274.

Moore, Jr. T. C., Rabinowitz, P. D., et al., 1984, Initial Reports of the Deep Sea Drilling Project, Volume 74: Washington, D.C., U.S. Government Printing Office, p. 1-894.

Norris, R. D., Bice, K. L., Magno, E. A., Wilson, P.A., and Bralower, T.,2002, Jiggling the tropical thermostat in the Cretaceous hot house. Geology (in press)

Norris, R.D. and Wilson, P.A., 1998, Low-latitude sea-surface temperatures for the midCretaceous and the evolution of planktic foraminifera: Geology, v. 26, p. 823-826.

Sellwood, B.W., Price, G.D., and Valdes, P.J., 1994, Cooler estimates of Cretaceous temperatures: Nature, v. 370, p. 453-455.

Sliter, W.V., 1977, Cretaceous foraminifers from the southwestern Atlantic Ocean, Leg 36, Deep Sea Drilling Project, Barker, P. F., Dalziel, I. W. D., et al., Initial Reports of the Deep Sea Drilling Project, Volume 36: Washington, D.C., U. S. Government Printing Office, p. 519-573.

Thomas, E., 1990, Late Cretaceous through Neogene deep-sea benthic foraminifers (Maud Rise, Weddell Sea, Antarctica), Barker, P. F., Kennett, J. P., et al., Proceedings of the Ocean Drilling Program, Scientific Results, Volume 113: College Station, TX, Ocean Drilling Program, p. 571-594.

Tucholke, B.E., Vogt, P.R., et al., 1979, Initial Reports of the Deep Sea Drilling Project, Volume 44: Washington, D.C., U.S. Government Printing Office, p. 1-1115. 